\title{
THE FAULTY RESISTOR PROBLEMS AND THE INVERSE SOURCE PROBLEMS FOR RECTANGULAR ELECTRICAL NETWORKS
}

\author{
Younghun Mun
}

\begin{abstract}
This paper ultimately aims to develop noninvasive techniques to identify the inside of a given electrical network. Based on the theory of the partial differentiation equations and mathematical modeling, this paper devises the algorithms to find the locations of possible abnormalities. To ensure the certainty of the algorithms, this study restricted the forms of the network and the number of abnormalities, rendering it easy to prove the uniqueness of the position of the abnormalities.
\end{abstract}

\section{Introduction}

In the routine of our lives, we are surrounded by numerous networks from simple electric bulbs to computer networks. However, even the simplest electrical network is too complex to examine with naked eyes; even a tiny DRAM contains millions of circuits. Therefore, if a problem occurs in an electrical network within its structure, it is almost impossible to observe what is wrong. Then it seems to be quite natural to raise a question how the problematic part can be detected by looking over the outer measurement. This paper aims to examine the algorithms to find out the location of possible sources and erroneous edges in an electrical network by a noninvasive method.

The main problems dealt with in this study are the Faulty Resistor Problems, abbreviated as FRP, and Inverse Source Problems, abbreviated as ISP. To be precise, the FRP focus on the algorithm of finding out the exact position of possible erroneous edges, using the electrical potentials and currents measured on boundaries. On the other hand, a source in an electrical network is a node where the extraordinary current flows into or out of the network. The ISP focus on the algorithm to find out the exact positions of the possible sources, using the electrical currents measured on the boundary after applying electrical potentials and currents on the boundary. On both the FRP and the ISP, we

Received November 11, 2008.

2000 Mathematics Subject Classification. Primary 94C12; Secondary 31A25.

Key words and phrases. electrical networks, inverse conductivity problem, inverse source problem, discrete Laplacian. 
adopt the techniques used in the theory of partial differential equations and their inverse problems.

The FRP and the ISP are not, in general, easily solved in every electrical network structure; even the uniqueness cannot be ensured in some electrical networks. Therefore, for simplicity, this study restricts these problems only in rectangular networks, which have the shape of rectangles with boundaries. Only few books or papers (for example, $[2,3]$ ) deal with these kinds of problems and it seems that there is no results so far which deals with full version of algorithm of identifying the erroneous edge or the source. The main objective of this paper is to provide algorithms which identify either the position of the erroneous edge or the source in the rectangular network, by assuming that there is at most one erroneous edge or at most one source therein.

\section{Preliminaries}

By a graph $G=G(V, E)$ we mean a finite set $V$ of verties (or nodes) with a set $E$ of two-element subsets of $V$ (whose elements are called edges). As conventionally used, we denote either $x \in V$ or $x \in G$ the fact that $x$ is a vertex in $\mathrm{G}$.

A graph $G$ is said to be simple if it has neither multiple edges nor loops, and $\mathrm{G}$ is said to be connected if for every pair of vertices $x$ and $y$ there exist a sequence of vertices $x=x_{0} \sim x_{1} \sim x_{2} \sim \cdots \sim x_{n-1} \sim x_{n}=y$ such that $x_{j-1}$ and $x_{j}$ are connected by an edge (termed adjacent) for $j=1,2, \ldots, n$, where $x \sim y$ means that two vertices $x$ and $y$ are connected (adjacent) by an edge in E.

A weighted (undirected) graph is a graph $G(V, E)$ associated with a weight function $\omega: V \times V \rightarrow[0, \infty)$ satisfying

(i) $\omega(x, y)=\omega(y, x), \quad x, y \in V$

(ii) $\omega(x, y)=0$ if and only if $\{x, y\} \notin E$.

Here, $\{x, y\}$ denotes the edge connecting the vertices $x$ and $y$.

From now on, the term network denotes a finite, connected, simple and weighted graph. For a given network $G(V, E, \omega)$, the $\omega$-Laplacian $\Delta_{\omega}$ of a function $f: V \rightarrow \mathbf{R}$ on $G$ is defined by

$$
\Delta_{\omega} f(x):=\sum_{y \in V}[f(y)-f(x)] \omega(x, y), \quad x \in V .
$$

A network $S=S\left(V^{\prime}, E^{\prime}\right)$ is said to be a subnetwork of $G(V, E)$ if $V^{\prime} \subset V$ and $E^{\prime} \subset E$. If $E^{\prime}$ consists of all the edges from $E$ which connect the nodes of $V^{\prime}$ in its host network $G$, then $S$ is called an induced subnetwork. For a induced subnetwork $S$ of a network $G=G(V, E)$, the boundary $\partial S$ of $S$ is the set of all nodes $z \in V$ not in $S$ but adjacent to some nodes in $S$, i.e., $\partial S:=\{z \in V \mid z \sim y$ for some $y \in S\}$. Also, by $\bar{S}$ we denote a network whose nodes and edges are in $S$ and nodes in $\partial S$. The nodes in $S$ is called interior nodes of $\bar{S}$. For a given network $\bar{S}$ with weight $\omega$, the normal derivative $\frac{\partial f}{\partial_{\omega} n}(z)$ 
at $z \in \partial S$ is defined to be

$$
\frac{\partial f}{\partial_{\omega} n}(z):=\sum_{y \in S}[f(z)-f(y)] \omega(z, y), \quad z \in \partial S .
$$

A rectangular network is the network whose interior nodes are arranged as a rectangular part of the 2 dimensional integral lattice and each boundary is branched from the nodes of the outside. By $I \times J$ network, $I, J \in \mathbf{N}$, we mean a rectangular network consisting of $I$ boundary nodes of horizontal side and $J$ boundary nodes of vertical side. For example, $6 \times 4$ network is seen in the Figure 1. Every node within the dotted rectangle is the interior node, and the others with the labels are boundary nodes.

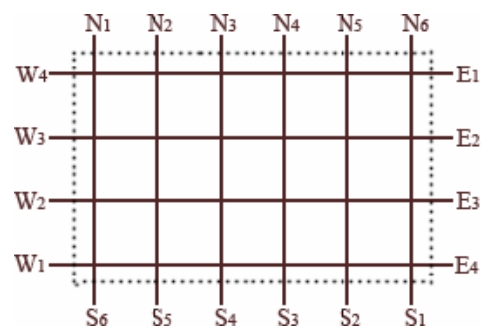

Figure 1.

For more details for the notations, we refer to [1].

Remark 2.1. If one considers an electrical network with potential $f(x)$ at each node $x$, it can be easily seen from Ohm's law that $\Delta_{\omega} f(x)$ represents the (total) current at $x$ induced by the potentials $f(y)$ given at the nodes $y$ neighboring to $x$. Here, the weight $\omega$ plays a role of conductivity on edges between two nodes. Kirchhoff's law is then mathematically modeled by $\Delta_{\omega} f(x)=0$.

Remark 2.2. Suppose that there is an electrical network with boundary where one can impose potential $\sigma$ freely on its boundary nodes. Then since the interior nodes satisfy Kirchhoff's law, the phenomenon is mathematically modeled by

$$
\begin{cases}-\Delta_{\omega} f(x)=0, & x \in S, \\ f(z)=\sigma(z), & z \in \partial S .\end{cases}
$$

Moreover, the currents $\psi$ on boundary is the normal derivative $\frac{\partial f}{\partial_{\omega} n}$ on boundary.

\section{The faulty resistor problems}

Suppose that an electrical network is perturbed so that there occurs some edges whose conductances differ from the normal ones. Such edges are said to be the erroneous edges. The Faulty Resistor Problems are to identify erroneous edges from boundary observations. The study in this section provides an efficient algorithm to solve the FRP for rectangular networks under the assumption that the network is supposed to have at most one erroneous edge. 
The general case that there exists more than two erroneous edges is left as a future work.

\subsection{Mixed boundary value problems}

For boundary nodes $p$ and $q$, a path from $p$ to $q$ is defined as a series of edges and nodes which are connected as

$$
p \sim r_{1} \sim r_{2} \sim \cdots \sim r_{k} \sim q
$$

for some distinct interior nodes $r_{1}, r_{2}, \ldots, r_{k}$ where $r_{i}$ and $r_{i+1}$ are adjacent by an edge for $i=1,2, \ldots, k-1$. For the two disjoint sets of boundary nodes $P=\left(p_{1}, p_{2}, \ldots, p_{k}\right)$ and $Q=\left(q_{1}, q_{2}, \ldots, q_{k}\right)$, we say that $P$ and $Q$ are in $g o o d$ connection if there are $k$ disjoint paths which join $p_{i}$ and $q_{i}$ as seen in the Figure 2.

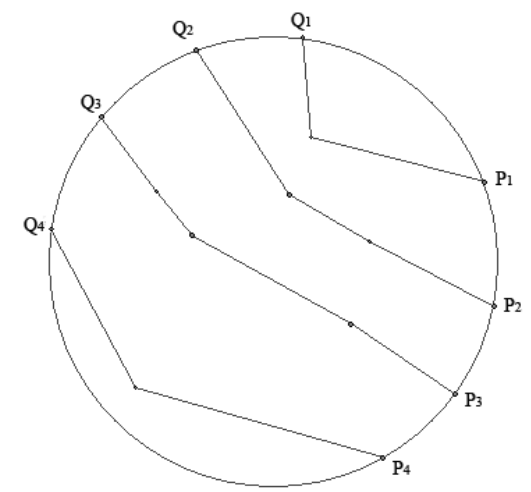

Figure 2.

The following mixed boundary value problem was proved in [3].

Theorem 3.1 ([3], Mixed Boundary Value Problems). Let $\bar{S}$ be a network with boundary where $\partial S \neq \emptyset, \omega$ be the weight on $\bar{S}$. Suppose that $\partial S=T_{1} \cup T_{2} \cup T_{3}$ where $T_{j}$ are mutually disjoint and $\sigma: T_{1} \cup T_{2} \rightarrow \mathbf{R}, j=1,2$ and $\psi: T_{2} \rightarrow \mathbf{R}$ are given. If $T_{2}$ and $T_{3}$ are in good connection, then the solution $f: \bar{S} \rightarrow \mathbf{R}$ of the following mixed boundary value problem

$$
\begin{cases}-\Delta_{\omega} f(x)=0, & x \in S, \\ f(z)=\sigma(z), & z \in T_{1} \cup T_{2}, \\ \frac{\partial f}{\partial_{\omega} n}(z)=\psi(z), & z \in T_{2}\end{cases}
$$

is uniquely determined.

\subsection{Naming the boundaries}

For a given $I \times J$ network, from the upper left corner, we call the first northern boundary nodes $N_{1}$ and the second $N_{2}$, and so on. Boundary nodes of other sides, east, south and west, can be also named $E_{j}, S_{x}$ and $W_{j}$ respectively, 
with clockwise increasing index numbers $j . \quad N, E, S$ and $W$ denote the set of northern, eastern, southern and western boundary nodes, respectively.

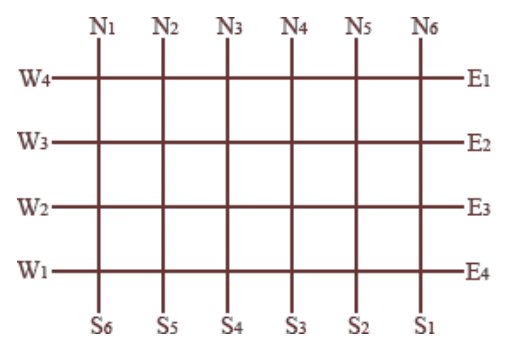

Figure 3.

Throughout this paper, without loss of the generality, we assume $I>J$ for $I \times J$ networks.

\subsection{Input and response}

The following result is obtained immediately from Theorem 3.1.

Theorem 3.2. For a given $I \times J$ network $\bar{S}$, let $\omega_{1}$ and $\omega_{2}$ be weights on $\bar{S}$, $\sigma: N \cup E \cup S \rightarrow \mathbf{R}$ and $\psi: E \rightarrow \mathbf{R}$ be given. Suppose $f_{1}$ and $f_{2}: \bar{S} \rightarrow \mathbf{R}$ satisfy

$$
\begin{cases}-\Delta_{\omega_{j}} f_{j}(x)=0, & x \in S, \\ f_{j}(z)=\sigma(z), & z \in N \cup E \cup S, \\ \frac{\partial f_{j}}{\partial_{\omega} n}(z)=\psi(z), & z \in E\end{cases}
$$

for $j=1,2$. If $f_{1} \neq f_{2}$ on $W$, then $\omega_{1} \neq \omega_{2}$.

Proof. It suffices to show that $f_{j}: \bar{S} \rightarrow \mathbf{R}$ satisfying (1) is uniquely determined, which is easily seen from the fact that $E$ and $W$ are in good connection.

Remark 3.3. From the proof of the above theorem, one can easily see that the theorem still holds after one rotate the network so that $N, E, S$ and $W$ changes to $E, S, W$ and $N$, respectively.

Remark 3.4. The converse of Theorem 3.2 does not always hold. For example, suppose that the boundary condition is given as follows:

$\sigma: N \cup E \cup S \rightarrow \mathbf{R}$ is defined as

$$
\sigma(z)= \begin{cases}1, & z=N_{1} \\ 0, & \text { otherwise }\end{cases}
$$

and $\psi \equiv 0$ on $E$ (Figure 4 represents the boundary condition for $2 \times 3$ network). Since $\sigma(z)=\psi(z)=0$ at $z=E_{1}$, it is easy to see that $f_{j}(x)=0, j=1,2$ at the node $x$ adjacent to $E_{1}$. Using similar arguments, we obtain $f_{j}(x)=0$ on each interior nodes $x$, regardless of the weights $\omega_{j}, j=1,2$. Therefore, if the edge $\{x, y\}$ satisfying $\omega_{1}(x, y) \neq \omega_{2}(x, y)$ does not belong to the dotted path, then $f_{1}=f_{2}$ on $W$. 


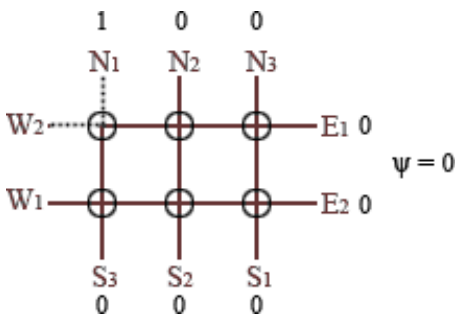

Figure 4.

Let us call the path diagonally connecting the nodes $N_{1}$ and $W_{J}$ the $N_{1}$-path. In Figure 4, for example, $N_{1}$-path consists the two dotted edges. It is noticeable that the arguments in Remark 3.4 implies the following important result.

Theorem 3.5. Let $\bar{S}$ be an $I \times J$ network. Suppose $f_{1}$ and $f_{2}: \bar{S} \rightarrow \mathbf{R}$ are the solutions of the equation (1) for $j=1,2$, where the boundary conditions are given the same as those in the above remark. If $f_{1} \neq f_{2}$ on $W$, then the edge $\{x, y\}$ satisfying $\omega_{1}(x, y) \neq \omega_{2}(x, y)$ lies in the $N_{1}$-path.

Similar to the $N_{1}$-path, we define the $N_{j}$-path as the path (left) diagonally connects from the node $N_{j}$ to the other boundary node. For example, in Figure 4 , the $N_{2}$-path consists the four edges connecting from the node $N_{2}$ to the node $W_{1}$. The $N_{3}$-path consists the 5 edges diagonally connects the node $N_{3}$ to the node $S_{3}$. Rotating clockwise, the $E_{j}$ and $S_{j}$-paths also can be defined using the same manner. For example, $E_{2}$-path in Figure 4 is the path (upper left) diagonally connects the node $E_{2}$ to the node $N_{2}$.

Observing the arguments in Remark 3.4 carefully, one can generalize Theorem 3.5 as follows.

Theorem 3.6. Let $\bar{S}$ be an $I \times J$ network and $j_{0} \in\{1,2, \ldots, I\}$ be given . Suppose $f_{1}$ and $f_{2}: \bar{S} \rightarrow \mathbf{R}$ are the solutions of the equations (1) for $j=1,2$, where the boundary conditions are given as

$$
\sigma(z)= \begin{cases}1, & z=N_{j}, \\ 0, & \text { otherwise }\end{cases}
$$

and $\psi \equiv 0$ on $E$. If $f_{1} \neq f_{2}$ on $W$, then the edge $\{x, y\}$ where $\omega_{1}(x, y) \neq$ $\omega_{2}(x, y)$ lies in the $\bigcup_{k=1}^{j} N_{k}$-path.

By rotating the network counterclockwise, one also obtain the following result.

Corollary 3.7. Let $\bar{S}$ be an $I \times J$ network and $j_{0}$ be given. Suppose $f_{1}$ and $f_{2}: \bar{S} \rightarrow \mathbf{R}$ are the solutions of the equations (1) for $j=1,2$, where the boundary conditions are given as

$$
\sigma(z)= \begin{cases}1, & z=E_{j}\left(\text { resp. } S_{j}\right) \\ 0, & \text { otherwise }\end{cases}
$$


and $\psi \equiv 0$ on $S$ (resp. $W)$. If $f_{1} \neq f_{2}$ on $N$ (resp. E), then the edge $\{x, y\}$ where $\omega_{1}(x, y) \neq \omega_{2}(x, y)$ lies in the $\bigcup_{k=1}^{j} E_{k}$-path (resp. $S_{k}$-path).

\subsection{An algorithm}

Suppose that an electrical rectangular network is perturbed that the conductivity of one edge is changed. Based on the results in the previous subsection, we provide an algorithm to detect the perturbed edge via boundary measurements.

For a boundary node $N_{j}, j=1,2, \ldots, I$, the $N_{j}$-experiment denotes the experiment imposing the potential $\sigma$ the same as that in (2) and the current $\psi \equiv 0$ on $E$ and then measuring the potential on $W$. Experiments on the other sides can be defined in the same manner by rotating $N_{j}$ experiment clockwise. Figure 5 and Figure 6 presents $N_{4}$ and $E_{3}$ experiments, respectively. When we obtain potentials of the nodes in $W$ from the experiment $N_{j}$, we call it the result of the $N_{j}$ experiment. If a network is perturbed so that there occurs an erroneous edge, some experiments may lead different results comparing with the results obtained from the same experiments performed on the normal network. Such a result is said to be an erroneous result.

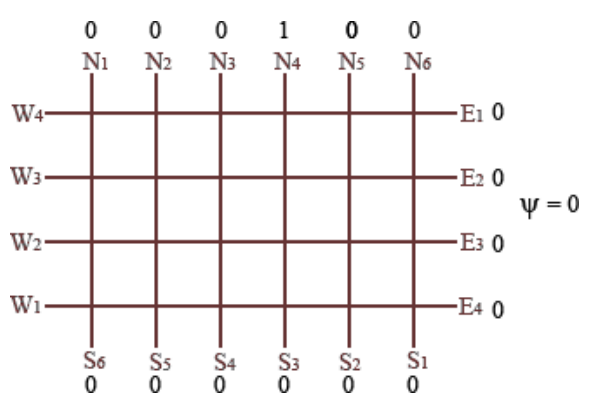

Figure 5 .

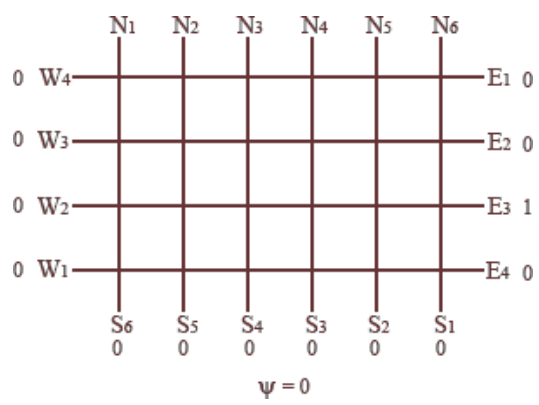

Figure 6.

Remark 3.8. On the other hand, we define experiments with slight modification from the experiments previously defined as follows:

as opposed to the previous case - imposing potential 0 on $N, E, S$ and current 0 on $E$ and observing the potentials on $W$ - we, in this time, impose potential 0 on $N, W, S$ and current 0 on $W$ and observe the potentials on $E$. To differentiate from the prior ones, we denote this kind of an experiment as $N_{j}^{\prime}, j=1,2, \ldots$.

In $I \times J$ network, at most $2(I+J)$ experiments should be performed to find out the exact position of the erroneous edge. We suppose that sets of normal results - the result obtained from the experiment which was performed before the perturbation occurred - are given beforehand. Following the steps proposed below, we can always and efficiently identify the erroneous edge via boundary measurements. 


\section{[STEP1]}

First, we perform the $N_{1}$ experiment, and simultaneously compare the results with those of normal network. If certain experiment leads an erroneous result, we call such an experiment an erroneous experiment for convenience. In the first performance, there are two possibilities - $N_{1}$ experiment is an erroneous experiment or not. If $N_{1}$ experiment is an erroneous experiment, we conclude from Theorem 3.6 that one of two edges in the $N_{1}$ path has an error and move on to STEP3. If $N_{1}$ experiment is not an erroneous experiment, however, we perform $N_{2}$ experiment. Again there are two possibilities: $N_{2}$ experiment may be erroneous or not. If $N_{2}$ experiment is an erroneous experiment, it follows from Theorem 3.6 and the fact that $N_{1}$ experiment is not an erroneous experiment, we can be sure that the erroneous edge is on the $N_{2}$-path; and then, move on to STEP3. If $N_{2}$ experiment is still normal, perform the same experiments on the rest boundary nodes in $N$ until we meet an erroneous experiment. After finding an erroneous experiment at $N_{j}$, conclude that the erroneous edge lies on $N_{j}$-path and move on to the STEP3. If no erroneous experiment is found even after finishing every experiment on the $N$, be sure that the erroneous edge never lies on the $N_{j}$-paths for each edge $N_{j}$ in $N$, and move on to STEP2.

\section{[STEP2]}

Repeat the STEP1 to the side $W$ and side $S$ with a slight change - we perform only $S_{1}, S_{2}, \ldots, S_{J}$ experiments, without performing $S_{J}, S_{J+1}, \ldots, S_{I}$ - until we get a boundary node $A$ which leads an erroneous experiment (See Remark 3.9 below).

\section{[STEP3]}

It is easy to see that during STEP1 and STEP2, one meets a boundary node $z$, which cause an erroneous experiment. This implies the fact that the erroneous edge lies in the $z$-path.

We now perform experiments on the side $W$. If we meet an erroneous experiment, move on the STEP4. Otherwise perform $N_{j}^{\prime}$ experiments in the opposite direction starting from $j=J$ to $j=1$ until we meet an erroneous experiment.

\section{[STEP4]}

During STEP 1, 2, 3, we meet two boundary nodes $z$ and $z^{\prime}$ which cause erroneous experiments. Finally we identify the erroneous edge as the edge lies in $z$-path and $z^{\prime}$-path simultaneously.

Remark 3.9. Since the $N_{j}$-path and the $S_{I-J+1-j}$-path coincides, it follows from Theorem 3.6 that $S_{I-J+1-j}$ experiment is basically the same as $N_{j}$ experiment. Thus, to avoid repeating the same experiment, we do not carry out $S_{I-J+1}, S_{I-J+2}, \ldots, S_{I}$ experiments in STEP2. 


\subsection{An example}

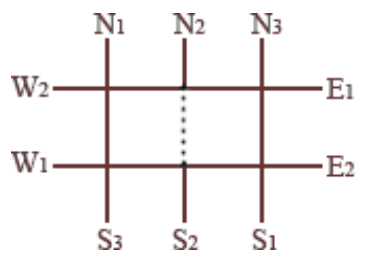

Figure 7.

Consider the $3 \times 2$ network given in the above figure. We suppose that the conductivity of every edge is given 1 , and calculate the normal results of experiments. The normal results are shown in the table below. Then, let us assume that the dotted edge in the Figure 7 is the erroneous edge, and measure results. Our task is to identify the erroneous edge only with outer measurements enumerated in the table below. During the course of checking from $N_{1}$ experiment to $N_{1}^{\prime}$ experiment, we stop if we obtain two erroneous experiments.

\section{TABLE 1}

\begin{tabular}{|l|l|l|l|}
\hline Experiment & Normal & Abnormal & Agreement \\
\hline$N_{1}$ & $0,-1$ & $0,-1$ & $O$ \\
\hline$N_{2}$ & $1,-4$ & $1,-4$ & $O$ \\
\hline$N_{3}$ & 8,16 & $13,-21$ & $X$ \\
\hline$E_{1}$ & $0,0,-1$ & $0,0,-1$ & $O$ \\
\hline$E_{2}$ & $0,1,-4$ & $0,1,-4$ & $O$ \\
\hline$S_{1}$ & 0,1 & 0,1 & $O$ \\
\hline$S_{2}$ & $1,-4$ & $1,-4$ & $O$ \\
\hline$W_{1}$ & $0,0,-1$ & $0,0,-1$ & $O$ \\
\hline$W_{2}$ & $0,1,-4$ & $0,1,-4$ & $O$ \\
\hline$N_{1}^{\prime}$ & 16,8 & $-21,13$ & $X$ \\
\hline
\end{tabular}

Based on the Table 1,we can find the first erroneous experiment in each side. It is observed that normal and abnormal results of the $N_{3}$ experiment differ and so does $N_{1}^{\prime}$ experiment. From this pair of first erroneous experiment, the erroneous edge can be uniquely determined: Where $N_{3}-S_{3}$ path and $N_{1}-S_{1}$ path meet is erroneous edge. As seen in the Figure 8, the $N_{3}-S_{3}$ path and $N_{1}-S_{1}$ path meets at the circled edge which is the same as we first assume.

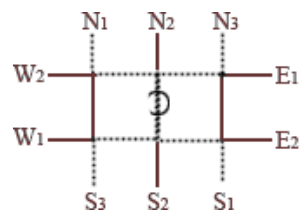

Figure 8 . 


\section{Inverse source problems}

In electrical network $\bar{S}$, by Kirchhoff's theorem, the interior nodes always have zero net current. But sometimes we have to consider an abnormal network, which contains some nodes that have nonzero net currents, which is mathematically modeled as

$$
\begin{cases}-\Delta_{\omega} f(x)=g(x), & x \in S, \\ f(z)=\sigma(z), & z \in \partial S,\end{cases}
$$

where $g(x) \neq 0$ if and only if $x \in\left\{x_{0}, x_{1}, \ldots, x_{n}\right\} \subset S$. Such a node $x_{j}$ is said to be the source.

Remark 4.1. It is known that the equation (3) always has unique solution (see, for example, [1]).

The main objective of this section is to provide an algorithm to detect a source of a network by giving potentials on boundary nodes and then measuring their current, when the network is known to have only one source in its interior.

First of all, we consider the following well-known result.

Theorem 4.2 ([2], Minimum and Maximum principle). Let a network $G=$ $G(V, E)$ with weight $\omega$ be given. Suppose $V_{1}$ and $V_{2}$ are two disjoint sets of nodes satisfying $V=V_{1} \cup V_{2}$ and $f: V \rightarrow \mathbf{R}$ is a function satisfying

$$
\Delta_{\omega} f(x)=0, \quad x \in V_{1} .
$$

If $f$ has either a minimum or maximum value in $V_{1}$, then $f$ is constant in $V_{1} \cup V_{2}$.

By virtue of the above theorem, the following result is easily obtained.

Corollary 4.3. Let a network $\bar{S}$ with weight $\omega$ be given. For a given node $x_{0} \in S$, suppose that $g: S \rightarrow \mathbf{R}$ is a function satisfying $g(x) \neq 0$ if and only if $x=x_{0}$. If $f$ is the nonconstant solution of the equation

$$
\begin{cases}-\Delta_{\omega} f(x)=g(x), & x \in S, \\ f(z)=\sigma(z), & z \in \partial S,\end{cases}
$$

then $f$ has minimum and maximum value in $\partial S \cup\left\{x_{0}\right\}$.

Based on the result in the above corollary, we provide an algorithm to detect source term in the rectangular electrical network under the assumption that the source occurred at only one interior node.

Suppose we have a rectangular electrical network $\bar{S}$ with weight (conductance) $\omega$, which is known to have a source term in one interior node.

\section{[STEP 1]}

Impose potential $f(z)=0$ to all the boundaries and then, measure boundary currents $\psi(z)=\frac{\partial f}{\partial_{\omega} n}(z)$. Comparing the values $f$ and $\psi$ at $z \in \partial S$, we can calculate the potential $f(x)$ of each node $x$ which lies right below the boundary 
nodes. In the Figure 9, such nodes are denoted to be surrounded by circles. We call the set of the nodes the shell.

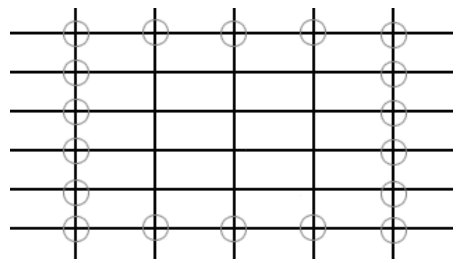

Figure 9 .

Remark 4.4. By virtue of Corollary 4.3 and the fact that zero potential is imposed to all the boundaries in STEP1, we can say that if the source has a positive (resp. negative) value, then every other interior node has a positive (resp. negative) value. Without loss of generality, we assume the source has the positive value of potential.

\section{[STEP2]}

Choose the node $v_{1}$ where the potential $f\left(v_{1}\right)$ is the largest potential $f(x)$ among the nodes $x$ in the present shell (see Figure 10). There are two possibilities : $v_{1}$ is unique or not. In the former case, go to STEP 3.

If there are more than two nodes $v_{1,1}, v_{1,2}, \ldots$ which have the largest potential simultaneously, then by Corollary 4.3 and the assumption that the network contains only one source term, we conclude that $v_{1, j}$ is not a source for each $j$. Then since $\Delta_{\omega} f(x)=0$ for each $x$ in the present shell, we can calculate every potential $f(x)$ where $x$ is the node in the next shell. Now repeat STEP2 for this new shell.

\section{[STEP3]}

By Corollary 4.3, every node $x$ in the present shell except $v_{1}$ is now guaranteed not to be a source and so $\Delta_{\omega} f(x)=0$ which means current zero at $x$. Based on this fact, we then calculate the potentials $f(x)$ of nodes $x$ in the next shell (the shell right inside the present shell), except the node located right below $v_{1}$. Compare the magnitude of the potential $f(x)$ of each node $x$ in the shell, except for the node right below $v_{1}$, with that of the node $v_{1}$. Then there are two possibilities $-v_{1}$ is the node with the largest potential or not.

In the former case, go to STEP4.

In the latter case, $v_{1}$ cannot be the source, because there is a node whose potential is bigger than that of $v_{1}$. Thus, from the fact that the current on $v_{1}$ is zero, potential on the node right below $v_{1}$ (let us call it $v_{1}^{\prime}$ ) can be calculated (see Figure 11), which means we now know every potential on the nodes in the second shell. Choose the node which has the larger potential $f(x)$ among the shell. Now, we newly call it $v_{1}$ instead of the former $v_{1}$ and then repeat STEP3 again. 


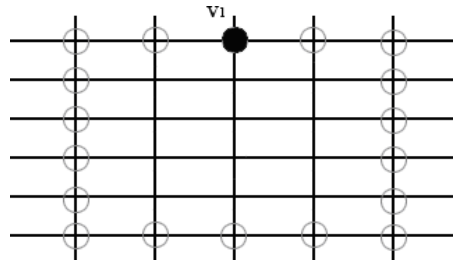

Figure 10 .

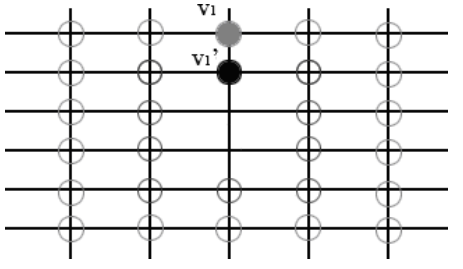

Figure 11.

If such $v_{1}$ lies in the smallest shell, we can easily calculate potentials of all of the nodes in this network. Then we finally determine the source as the node of the largest potential and quit the algorithm.

\section{[STEP4]}

Let us start with $n=1$. Since every node in the shell except the node $v_{n+1}$ located right below $v_{n}$ is guaranteed not to be a source, we can calculate the potential on each of the node in the next shell except the node $v_{n+2}$ located right below $v_{n+1}$ (see Figure 16). Compare the magnitude of the potential of each node in the shell except $v_{n+2}$ with that of $v_{1}$.

Repeat this process for $n=1,2, \ldots, K$ till we find a node in $(K+2)$-th shell whose potential is larger than that of $v_{1}$. After we find such a node, we go to STEP5.

If such a node is not found even in the smallest shell, it is an easy matter to calculate potentials of all of the nodes in this network. Then we finally determine the source as the node of the largest potential and quit the algorithm.

\section{[STEP5]}

Let us start with $n=1$. Since $v_{n}$ is determined not to be a source, we can calculate the potential on $v_{n+1}$. Compare the potential of each node in the $(K+2)$-th shell except $v_{K+1}$ with that of $v_{n+1}$. Repeat this process for $n=1,2, \ldots, K$ till the node $v_{n+1}$ has the largest potential comparing with those of the nodes in the $(K+2)$-th shell. After we find such a node $v_{n+1}$, we newly call it $v_{1}$ and then go back to STEP3 (see Figure 12).

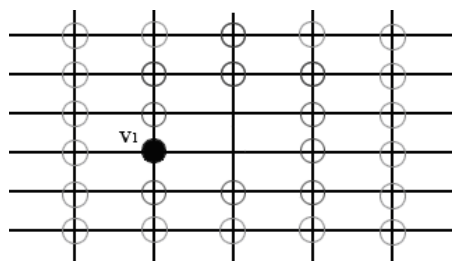

Figure 12.

If such a node $v_{n}$ is not found during the process $n=1,2, \ldots, K$, we conclude $v_{1}, \ldots, v_{K+1}$ are not a source and so, from the fact that their currents are zero, we calculate the potential on $v_{K+2}$. Choose the node with the largest potential among the nodes on $(K+2)$-th shell, call it newly $v_{1}$, and go back to STEP3. 
Acknowledgement. The author would like to express his sincere gratitude to professor Soon-Yeong Chung for his valuable comments during the seminars on Research and Education program.

\section{References}

[1] S.-Y. Chung and C. A. Berenstein, w-harmonic functions and inverse conductivity problems on networks, SIAM J. Appl. Math. 65 (2002), no. 4, 1039-1046.

[2] E. Curtis and J. Morrow, The Dirichlet to Neumann map for a resistor network, SIAM J. Appl. Math. 51 (1991), no. 4, 1011-1029.

[3] _ Inverse Problems for Electrical Networks, World Scientific, 2000.

Korea Minjok Leadership Academy

GANGWON-Do 225-823, KorEA

E-mail address: younghoon514@gmail.com 